\title{
Glucocorticoids upregulate CD40 ligand expression and induce CD40L-dependent immunoglobulin isotype switching
}

\author{
Haifa H. Jabara, Scott R. Brodeur, and Raif S. Geha \\ Division of Immunology, Children's Hospital and Department of Pediatrics, Harvard Medical School, \\ Boston, Massachusetts, USA
}

Address correspondence to: Raif S. Geha, Enders 8, Division of Immunology, 300 Longwood Avenue, Children's Hospital, Boston, Massachusetts 02115, USA. Phone: (617) 355-7603; Fax: (617) 355-8205; E-mail: geha@a1.tch.harvard.edu.

Received for publication April 25, 2000, and accepted in revised form December 18, 2000.

\begin{abstract}
IL-4 and CD40 ligation are essential for IgE synthesis by B cells. We have shown previously that hydrocortisone (HC) induces IgE synthesis in IL-4-stimulated human B cells. In this study we demonstrate that HC fails to induce IgE synthesis in B cells from CD40 ligand-deficient (CD40L-deficient) patients. Disruption of CD40L-CD40 interactions by soluble CD40-Ig fusion protein or anti-CD40L mAb blocked the capacity of $\mathrm{HC}$ to induce IgE synthesis in normal B cells. HC upregulated CD40L mRNA expression in PBMCs and surface expression of CD40L in PBMCs as well as in purified populations of T and $\mathrm{B}$ cells. Upregulation of CD40L mRNA in PBMCs occurred 3 hours after stimulation with HC and was inhibited by actinomycin D. Upregulation of CD40L mRNA and induction of IgE synthesis by HC were inhibited by the steroid hormone receptor antagonist RU-486. These results indicate that ligand-mediated activation of the glucocorticoid receptor upregulates CD40L expression in human lymphocytes.
\end{abstract}

J. Clin. Invest. 107:371-378 (2001).

\section{Introduction}

Ig isotype switching allows a single B-cell clone to produce $A b$ 's with the same fine specificity but different effector functions determined by the heavy-chain isotype. Two events are involved in B-cell isotype switching (1). The first event is the activation of transcription at a specific region of the Ig locus, thus determining isotype specificity. The second event is the activation of the recombination machinery, resulting in DNA switch recombination. Binding of the Th2-derived cytokines IL-4 or IL-13 to their receptor on the $\mathrm{B}$ cell induces transcription of the $\mathrm{C} \varepsilon$ heavy-chain gene locus $(2,3)$. This is amplified by engagement of the B-cell antigen by its ligand (CD40L, CD154), a member of the TNF superfamily that is expressed on activated T cells (4). CD40L and IL-4 synergize in activating deletional switch recombination to $\operatorname{IgE}(5)$. This has been shown recently to involve induction of the cytidine deaminase, activation-induced deaminase (AID) (6).

The importance of CD40L-CD40 interactions in isotype switching is illustrated by several observations. The mAb's directed against CD40 can replace $T$ cells in IL-4-driven isotype switching to IgE in vitro (7). Soluble human CD40 (sCD40) inhibits in vitro T cell-driven IL-4-dependent IgE synthesis (8). Patients with the $\mathrm{X}$-linked hyper-IgM (X-HIM) syndrome are deficient in CD40L and lack serum IgG, IgA, and IgE due to defective isotype switching in vivo (9). In addition, mice with targeted disruption of the CD40L or CD40 genes lack serum $\operatorname{IgE}$ and fail to undergo isotype switching after immunization with T cell-dependent antigens (10-12).

Steroid hormones are synthesized and secreted by endocrine cells. They enter target cells by simple or facilitated diffusion, and then bind to specific steroid hormone receptors (SHR) (13). The SHR superfamily represents the largest known family of transcription factors in eukaryotes. It includes the receptors for glucocorticoid (GR), mineralocorticoid, androgen, progesterone, and estrogen, as well as receptors for thyroid hormone, vitamin $\mathrm{D}$, retinoic acid, 9-cis retinoic acid, and a large number of "orphan receptors," the ligands for which are not known. SHRs exist in inactive forms either in the cytoplasm or nucleus, usually in complex with other proteins. Upon binding their respective hormonal ligands, SHRs undergo an activation step. In the case of GR, this involves dissociation from chaperones followed by nuclear translocation, binding a DNA glucocorticoid-response element (GRE), and activation of the transcription of a cis-linked gene. In addition to its role in the activation of gene transcription by GRE binding, ligand-bound GR may repress gene induction by binding to a negative GRE or by binding to and interference with other transcription factors, e.g., AP-1 $(14,15)$. GR also interacts and cooperates positively with transcription factors such as STAT3, STAT5, OCT, and, in the case of the myf5 gene, even with AP-1 (16-20).

We and others have shown previously that the glucocorticoids (GCs) hydrocortisone (HC) and dexam- 
ethasone (DM), but not mineralocorticoids or sex steroids, induce in the presence of IL-4 IgE synthesis by human $B$ cells $(21,22)$. This was shown to involve deletional switch recombination (23). The mechanism of induction of $\operatorname{IgE}$ isotype switching by $\mathrm{HC}$ is unknown. We here show that induction of $\operatorname{IgE}$ isotype switching by $\mathrm{HC}$ is dependent on CD40L and that HC upregulates CD40L mRNA in human PBMCs and surface-protein expression on PBMCs and on purified $\mathrm{T}$ and $\mathrm{B}$ lymphocytes.

\section{Methods}

Patients. Five patients with X-HIM syndrome were studied. They included one set of brothers. The mutations in CD40L were identified in four of these patients as follows: One patient had a $\mathrm{C} \rightarrow \mathrm{A}$ mutation at nucleotide 590 in the coding region and a deletion of the adjacent 591 nucleotide resulting in a frameshift with introduction of a stop codon after amino acid 240 (24). The second patient had a 58-bp deletion from nucleotides 289-346, which changes the reading frame (24). The two brothers had a 6-bp in-frame deletion (nucleotides 424-429) (25). After activation with phorbol myristate acetate (PMA) and ionomycin (IO), no CD40L expression was detected on the surface of the $T$ cells from any of the patients.

Cell preparation. PBMCs were isolated from heparinized blood obtained from patients with X-HIM syndrome or normal nonatopic donors by density-gradient centrifugation on Ficoll Hypaque (Amersham Pharmacia Biotech, Piscataway, New Jersey, USA) (7). Cells were then washed and resuspended in RPMI-1640 containing 10\% heat-inactivated FCS (Hyclone Laboratories, Logan, Utah, USA), $2 \mathrm{mM}$ L-glutamine, $50 \mu \mathrm{g} / \mathrm{ml}$ streptomycin, and $100 \mathrm{U} / \mathrm{ml}$ penicillin (Life Technologies Inc., Rockville, Maryland, USA) (complete medium). Purified B-cell populations were isolated from PBMCs by positive sorting with Dynabeads conjugated to $\mathrm{CD} 19$, while $\mathrm{T}$ cells were purified by negative sorting using the Dynal T-cell negative isolation kit according to the manufacturer's instructions (Dynal Inc., Lake Success, New York, USA). FACS analysis of the resultant purified cells showed that B cells were more than 99\% $\mathrm{CD}_{19}{ }^{+}$and $\mathrm{T}$ cells were more than $96 \% \mathrm{CD}^{+}$.

Cell cultures. For IgE production, PBMCs $\left(1.5 \times 10^{6}\right.$ cells $/ \mathrm{ml}$ ) were cultured in complete medium in the presence or absence of IL-4 (100 U/ml; DNAX Research Institute, Palo Alto, California, USA), plus either anti-CD40 mAb $626.1(5 \mu \mathrm{g} / \mathrm{ml}$; a kind gift of S.M. Fu, University of Virginia, Charlottesville, Virginia, USA), or HC $\left(10^{-6} \mathrm{M}\right.$; Sigma Chemical Co., St. Louis, Missouri, USA). After 14 days, supernatants were harvested and assessed for their IgE content. For Epstein-Barr virus-stimulated (EBV-stimulated) cultures, cells $\left(2 \times 10^{6}\right.$ cells $\left./ \mathrm{ml}\right)$ were suspended in complete medium plus supernatant from the marmoset B95-8 cell line (1:1 ratio; American Type Culture Collection, Rockville, Maryland, USA) in the presence or absence of IL-4. Every 3-4 days, half the culture super- natant was replaced by new complete medium with or without IL-4 as described previously (26). Supernatants were assessed for their IgE content after 21-24 days. In certain experiments, actinomycin D (Calbiochem), RU-486 (a kind gift of Katia Karalis, Children's Hospital, Boston, Massachusetts, USA), antiCD40L mAb (5c8; a kind gift of Chris Benjamin, Biogen Inc., Cambridge, Massachusetts, USA), or sCD40-IgG and sCD44-IgG as described (27), were added to the cultures.

IgE measurement. Levels of IgE in culture supernatants were measured using ELISA. Microtiter plates (Maxisorb; Nunc, Rochester, New York, USA) were coated overnight at $4^{\circ} \mathrm{C}$ with $50 \mu \mathrm{l} /$ well of anti-human IgE mAb's 7.12 and 4.15 (a kind gift of Andrew Saxon, University of California-Los Angeles School of Medicine, Los Angeles, California, USA) at $2 \mu \mathrm{g} / \mathrm{ml}$ each in $0.1 \mathrm{M}$ $\mathrm{NaHCO}_{3}$ buffer $\mathrm{pH}$ 9.0. After washing with PBS containing $0.05 \%$ Tween 20 , wells were blocked for 4 hours at room temperature with $2 \%$ dry milk in PBS containing $0.01 \%$ sodium azide. Culture supernatants or purified IgE standards (Amersham Pharmacia Biotech) were added $50 \mu \mathrm{l} /$ well to washed plates and kept in a humidified chamber overnight at $4^{\circ} \mathrm{C}$. Plates were washed and $50 \mu \mathrm{l}$ of 1:1,000 diluted horseradish peroxidase-labeled (HRP-labeled) rabbit anti-human $\operatorname{IgE}$ (DAKO Corp., Carpinteria, California, USA) was added for 4-6 hours at room temperature. After washing, the plate was developed with $100 \mu \mathrm{l}$ of $o$-phenylenediamine dihydrochloride (OPD; Sigma Chemical Co.), and the reaction was stopped by adding $100 \mu \mathrm{l}$ of $10 \% \mathrm{H}_{2} \mathrm{SO}_{4}$ and read at $492 \mathrm{~nm}$ using an ELISA reader (LP 400; Sanofi Diagnostic Pasteur, Marnes-la-Coquette, France). Net IgE synthesis was calculated by subtracting the amount of $\operatorname{IgE}$ detected in the supernatants of cycloheximide-treated $(100 \mu \mathrm{g} / \mathrm{ml}$, Sigma Chemical Co.) cultures. The sensitivity of the assay was $0.3 \mathrm{ng} / \mathrm{ml}$.

RT-PCR for CD40L expression. Total RNA was extracted from cell cultures using TRIzol (Life Technologies Inc.) according to the manufacturer's instructions. Firststrand cDNA was synthesized from 2-5 $\mu \mathrm{g}$ of total cellular RNA as described previously (24). The cDNA was subjected to 30 cycles of amplification using a PTC-100 thermal cycler (MJ Research Inc., Waltham, Massachusetts, USA) and Taq polymerase (Roche Molecular Biochemicals, Indianapolis, Indiana, USA). The primers used were 5'-CACCTTCTCTGCCAGAAGATACCATTTCAA$3^{\prime}$ (sense) and 5'-TTATGAAGACTCCCAGCGTCACGT CCACCA-3' (antisense), which permitted the amplification of the entire coding region of human CD40L cDNA. After denaturation at $94^{\circ} \mathrm{C}$ for 5 minutes, the amplification conditions were as follows: 1 minute at $94^{\circ} \mathrm{C}$, annealing at $62^{\circ} \mathrm{C}$ for 1 minute, and extension at $72^{\circ} \mathrm{C}$ for 3 minutes. The PCR products were resolved on a $1.2 \%$ agarose gel. For equal loading, primers that amplify a 438-bp cDNA fragment of the housekeeping gene GAPDH were used as described previously (28). The intensity of the CD40L and GAPDH PCR products were assessed by densitometry and quantitated by using the NIH Image 
Table 1

$\mathrm{IL}-4+\mathrm{HC}$ induces IgE synthesis in normal B cells but not in B cells from CD40L-deficient patients

\begin{tabular}{lccccccc}
\hline \multicolumn{7}{c}{ Net IgE synthesis $(\mathrm{ng} / \mathrm{ml})$} \\
\cline { 2 - 6 } & Controls & \multicolumn{7}{c}{ Patients } \\
Stimulus & $(n=4)$ & 1 & 2 & 3 & 4 & 5 \\
Medium & $<0.3$ & $<0.3$ & $<0.3$ & $<0.3$ & $<0.3$ & $<0.3$ \\
IL-4 & $3.6 \pm 3.4$ & $<0.3$ & $<0.3$ & $<0.3$ & $<0.3$ & $<0.3$ \\
IL-4+anti-CD40 & $30.1 \pm 23.1$ & 2.7 & 15.1 & 9.8 & 7.1 & 8.8 \\
IL-4+HC & $49.9 \pm 9.0$ & $<0.3$ & $<0.3$ & $<0.3$ & $<0.3$ & $<0.3$ \\
IL-4+EBV & $23.9 \pm 8.9$ & 16.7 & 2.4 & 2.1 & ND & 19.7 \\
& & & & &
\end{tabular}

PBMCs from normal donors or from CD40L-deficient patients were stimulated with medium, IL-4 (100 U/ml) alone, IL-4+anti-CD40 mAb $(5 \mu \mathrm{g} / \mathrm{ml})$, or HC $\left(10^{-6} \mathrm{M}\right)$. EBV was added as a culture supernatant from B95-8 EBV infected cells as described in Methods. Culture supernatants were collected on day 14 and assessed for their IgE content by using ELISA. Net IgE was calculated as described in Methods. Patients' results shown represent the mean of duplicate cultures; values for controls represent the mean of four different donors. ND, not done.

program 1.62 (Wayne Rasband, NIH); CD40L mRNA expression was calculated as the ratio of the intensity of the CD40L band to the GAPDH band.

FACS analysis. Cells were cultured for 6 hours with medium, PMA (20 ng/ml; Sigma Chemical Co.) and IO $(0.5 \mu \mathrm{M}$; Calbiochem-Novabiochem Corp., San Diego, California, USA), or HC $\left(10^{-6} \mathrm{M}\right)$, The cells were then harvested, washed in PBS containing $2 \%$ BSA, 5\% FCS, and $0.005 \%$ sodium azide, and resuspended in $50 \mu \mathrm{l}$ of this buffer supplemented with human IgG $(300 \mu \mathrm{g} / \mathrm{ml})$ to block nonspecific binding. Staining of cells was performed either by the standard method or by the enzymatic amplification technique developed by Kaplan and Smith (29). In the standard method, the cells were stained with either anti-CD40L phycoerythrin (PE) $\mathrm{mAb}$ or isotype control (Ancell Corp., Bayport, Minnesota, USA), according to the manufacturer's instructions. For the amplification staining, cells were incubated with either biotinylated anti-CD40L or biotinylated mouse IgG1 control (Ancell Corp.), followed by an amplification step using the Enzymatic Amplification Staining kit (Flow-Amp Systems, Cleveland, Ohio, USA) according to the manufacturer's instructions. This amplification consists of sequential 10-minute incubations at room temperature with streptavidin-HRP, followed by biotinylated tyramide in the presence of $0.01 \%$ peroxide, with a washing step in between. As a detector reagent, streptavidin conjugated to CyChrome (SA-Cy5; BDPharMingen, San Diego, California, USA) was used. The cells were then washed, and a minimum of 10,000 total events were analyzed using a FACScalibur flow cytometer (BD Immunocytometry Systems, San Jose, California, USA).

\section{Results}

HC+IL-4 induces IgE isotype switching in normal B cells, but not in B cells from CD40L-deficient patients. We have shown previously that $\mathrm{HC}+\mathrm{IL}-4$ induces IgE synthesis in normal PBMCs and in highly purified normal B cells (21). The range of concentrations of $\mathrm{HC}$ that resulted in $\operatorname{IgE}$ isotype switching was $10^{-7} \mathrm{M}$ to $10^{-5} \mathrm{M}$ with an optimum at $10^{-6} \mathrm{M}$. To explore the role of CD40L in the induction, we examined the response of PBMCs from five patients with $\mathrm{X}$-HIM syndrome. Table 1 shows that $\mathrm{HC}+\mathrm{IL}-4$ failed to induce IgE isotype switching in PBMCs from all five patients. As reported previously (27), PBMCs from the CD40L-deficient patients failed to synthesize IgE in response to IL-4 alone, an event that is strictly dependent on CD40L-CD40 interactions. In contrast, anti-CD40+IL-4 induced IgE synthesis in PBMCs from all patients, confirming that there is no intrinsic defect in the ability of their B cells to undergo isotype switching. Furthermore, incubation with EBV+IL-4 also resulted in IgE isotype switching in B cells from all four CD40L-deficient patients tested. These results suggest that CD40L may be required for the induction of IgE isotype switching by HC+IL-4.

The failure of HC+IL-4 to induce IgE switching in $B$ cells from CD40L-deficient patients was not due to a difference in the sensitivity of lymphocytes from these patients to HC. Incubation of PBMCs from the patients with concentrations of HC that ranged from $10^{-5} \mathrm{M}$ to $10^{-8} \mathrm{M}$ in the presence of IL- 4 did not result in IgE synthesis (data not shown).

Induction of IgE isotype switching by HC+IL-4 is inhibited by $s C D 40$ and by anti-CD $40 \mathrm{~L} \mathrm{mAb}$. It could be argued that the failure of $\mathrm{B}$ cells from CD40L-deficient patients to respond to $\mathrm{HC}$ might simply reflect their development in an abnormal environment rather than a requirement for CD40L. To assess the role of CD40L in the induction of IgE synthesis by HC+IL-4 in normal B cells, we examined the effect of blocking CD40L-CD40 interactions. For this purpose, we used a soluble CD40-IgG fusion protein $(\mathrm{sCD} 40)$ and a mouse anti-human CD40L mAb (5c8), which have been shown previously to inhibit IL-4-driven CD40L-dependent IgE synthesis in PBMCs $(8,30)$. Figure 1a shows that sCD40-IgG, but not the control sCD44-IgG fusion protein, inhibits induction of IgE synthesis by HC+IL-4. The enhancement of $\operatorname{IgE}$ synthesis observed with sCD44 in the experiment presented was not reproducible, because it was not observed in two other experiments. Figure $1 \mathrm{~b}$
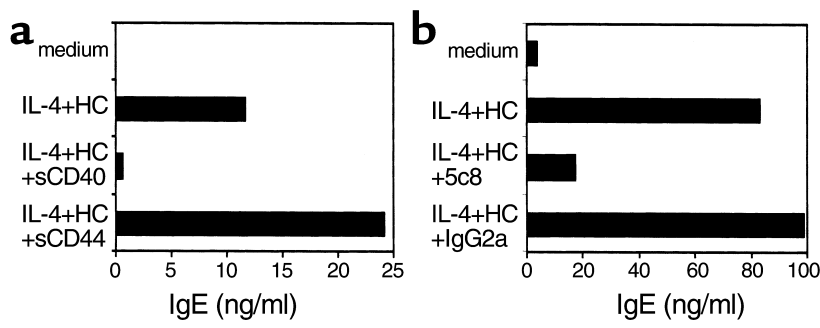

\section{Figure 1}

Inhibition of HC+IL-4 induction of IgE synthesis in PBMCs by sCD40-IgG and anti-CD40L mAb. PBMCs from normal donors were stimulated with $\mathrm{HC}\left(10^{-6} \mathrm{M}\right)$ plus IL-4 $(100 \mathrm{U} / \mathrm{ml})$ for 14 days in the absence or presence of (a) sCD40- $\operatorname{lgG}(5 \mu \mathrm{g} / \mathrm{ml})$ or control sCD44-IgG $(5 \mu \mathrm{g} / \mathrm{ml})$ and $(\mathbf{b})$ anti-CD40L mAb $(5 \mathrm{c} 8,0.1 \mu \mathrm{g} / \mathrm{ml})$ or isotype control lgG2a $(0.1 \mu \mathrm{g} / \mathrm{ml})$. Results shown are representative of three experiments. 


\section{Figure 2}

RT-PCR analysis of $\mathrm{HC}$ induction of CD40L expression. (a) PBMCs from a normal donor were stimulated for 3 hours with medium (-), HC $\left(10^{-6} \mathrm{M}\right)$, or PMA $(20 \mathrm{ng} / \mathrm{ml})$ plus IO $(0.5 \mu \mathrm{M})$. Equal amounts of total RNA $(5 \mu \mathrm{g})$ were reverse transcribed, and the cDNA was subjected to RT-PCR using primers that amplify the entire coding region of CD $40 \mathrm{~L}$ cDNA. Final PCR products were analyzed by agarose gel electrophoresis. For equal loading, primers that amplify a 438-bp cDNA fragment of the housekeeping gene GAPDH were used. (b) The effect of actinomycin D (Act D) on upregulation of CD40L mRNA expression by $\mathrm{HC}$ are shown. PBMCs were examined for CD40L mRNA expression as in $\mathbf{a}$ in the presence or absence of Act D $(10 \mu \mathrm{g} / \mathrm{ml})$. Values represent the mean plus or minus SE of the ratios of the intensity of the bands representing the RT-PCR products of $C D 40 \mathrm{~L}$ and $\mathrm{GAPDH}$ as assessed by densitometry in three experiments. Kinetics of CD40L mRNA expression in PBMCs stimulated with $\mathrm{HC}$ (c) or $\mathrm{PMA}+\mathrm{IO}$ (d) are shown. Values represent the mean $\pm \mathrm{SE}$ of the ratios of the intensity of the bands representing the RT-PCR products of CD40L and GAPDH in three experiments.

shows that mouse $\mathrm{mAb} 5 \mathrm{c} 8$, but not isotype control $\mathrm{mAb}$, inhibits HC+IL-4 induction of IgE synthesis. These results support the notion that induction of $\operatorname{IgE}$ isotype switching by $\mathrm{HC}+\mathrm{IL}-4$ is dependent on CD $40 \mathrm{~L}$.

$H C$ induces CD4OL $m R N A$ expression in buman PBMCs. The above results suggest that HC may upregulate CD40L expression. We first examined CD40L mRNA expression by RT-PCR in human PBMCs stimulated with HC. Cells treated with PMA+IO served as positive control. Figure 2a shows that unstimulated PBMCs express low levels of CD40L mRNA. Treatment with HC for 3 hours upregulated CD40L mRNA expression, but to a lesser extent than treatment for 3 hours with PMA+IO. In six experiments, HC stimulation for 3 hours induced a $4.9 \pm 2.5$-fold significant increase in CD40L mRNA expression as compared with unstimulated cells $(P<0.03$, one sample $t$ test $)$, while a $20.4 \pm 9.0$ fold increase was seen after stimulation with PMA $+\mathrm{IO}$. Similar results were obtained with DM, which has relatively more pure GC activity than HC (data not shown). Progesterone, which fails to induce IgE synthesis in IL-4-stimulated B cells (22), failed to upregulate CD40L mRNA expression (data not shown).

Upregulation of CD40L mRNA expression by HC may have resulted from enhanced transcription of the CD40L gene and/or from enhanced stabilization of pre-existing CD40L mRNA. To distinguish between these possibilities, we examined the effect of the transcription inhibitor actinomycin $\mathrm{D}$ on the upregulation of CD40L mRNA by HC. Figure $2 \mathrm{~b}$ shows that upregulation of CD 40L mRNA by HC was abolished by actinomycin $\mathrm{D}$, suggesting that it resulted from increased transcription of the CD40L gene. Furthermore, culture with actinomycin $\mathrm{D}$ for 3 hours abolished baseline expression of CD40L mRNA, which is consistent with the short half life for CD40L mRNA.

CD40L mRNA expression in T cells stimulated by $\mathrm{PMA}+\mathrm{IO}$ or via the TCR/CD3 is rapid and transient (31). Figure $2 c$ depicts the kinetics of upregulation of a

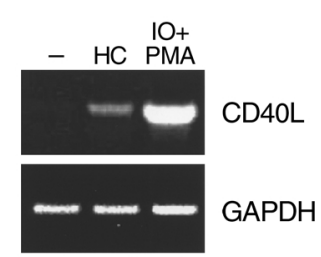

b
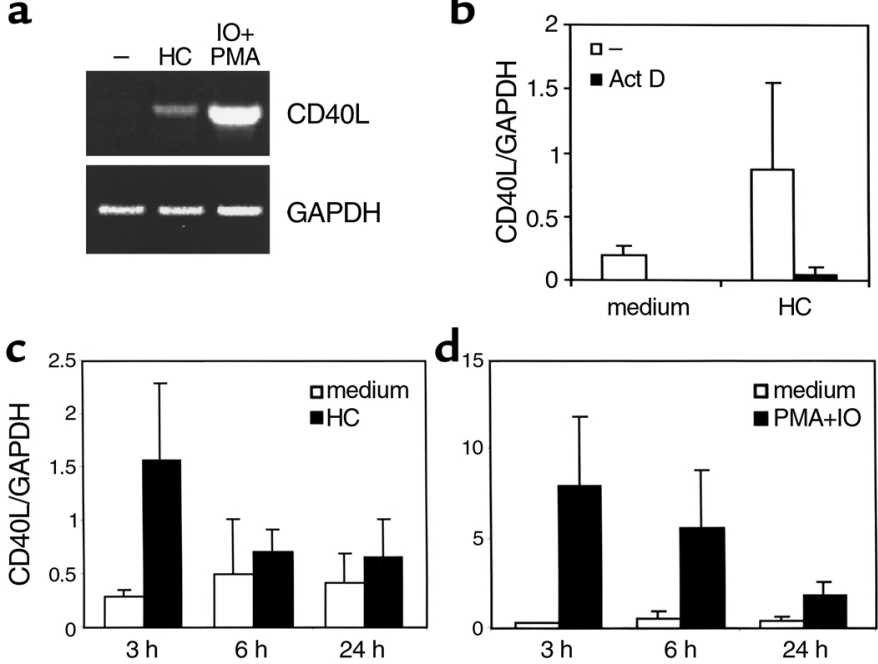

CD40L mRNA after stimulation of PBMCs. In three experiments, upregulation of CD $40 \mathrm{~L}$ mRNA expression occurred 3 hours after HC stimulation and subsided by 6 hours. In the same experiments, and as previously reported (31), upregulation of CD40L mRNA expression after stimulation with $\mathrm{PMA}+\mathrm{IO}$ peaked at 3 hours, was still evident at 6 hours, and subsided by 24 hours (Figure 2d).

HC induces $C D 40 L$ surface-protein expression in buman PBMCs and T and B cells. FACS analysis was used to examine whether $\mathrm{HC}$ also induces surface expression of CD40L. Standard staining with PE-conjugated antiCD $40 \mathrm{~L} \mathrm{mAb}$ revealed that $\mathrm{HC}$ induces a small, but reproducible, shift of the fluorescence intensity profile compared with unstimulated cells (data not shown). Enhanced flow cytometry using enzymatic amplification was subsequently used. Figure 3a shows that low expression of CD40L was detected on unstimulated normal PBMCs This was specific because, using the same technique, there was no detectable CD40L expression on either unstimulated or PMA+IO-stimulated PBMCs from a patient with X-HIM syndrome who had absent CD40L protein expression as determined by standard staining. Stimulation of normal PBMCs with HC resulted in an increase in the percentage of CD40L-expressing cells as well as in the mean fluorescence intensity (MFI) of CD40L staining. As predicted, CD40L was upregulated in PBMCs stimulated with PMA+IO as control.

The capacity of HC to induce CD40L expression in highly purified $\mathrm{T}$ - and B-cell populations was examined. $\mathrm{HC}$ induced CD40L expression in both purified normal human $\mathrm{T}$ - and B-cell subpopulations (Figure $3 \mathrm{~b}$ ). It should be noted that only unstimulated $T$ cells, but not B cells, expressed baseline CD40L. Furthermore, following stimulation with HC, the intensity of surface CD40L expression was higher on T cells than B cells.

Induction of IgE synthesis by HC involves the GR. Steroid hormones may exert activities independent of binding 
Figure 3

Upregulation of surface CD40L expression on PBMCs and $\mathrm{T}$ and $\mathrm{B}$ cells in response to $\mathrm{HC}$ stimulation. (a) PBMCs $\left(10^{6} / \mathrm{ml}\right)$ from normal donors or from patients with X-HIM syndrome were cultured for 6 hours with medium, HC $\left(10^{-6} \mathrm{M}\right)$, or PMA $(20 \mathrm{ng} / \mathrm{ml})$ plus IO $(0.5 \mu \mathrm{M})$. Cells were then washed and stained with biotin-conjugated antiCD40L mAb (solid line) or with biotin-conjugated isotype control (dashed line), using the enzymatic amplification method described in Methods, and were analyzed using FACS. Similar results were obtained in two experiments. (b) Purified T or B cells $\left(10^{6} / \mathrm{ml}\right)$ from a normal donor were cultured as in a with either medium or HC. Similar results were obtained in two experiments.
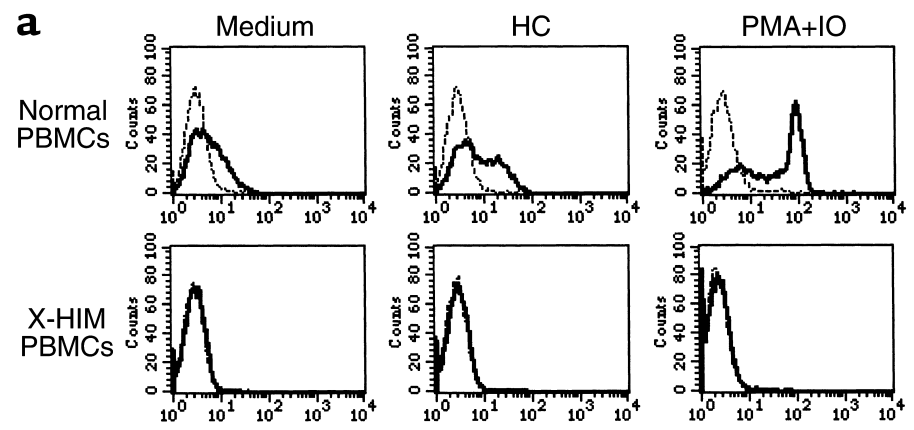

b
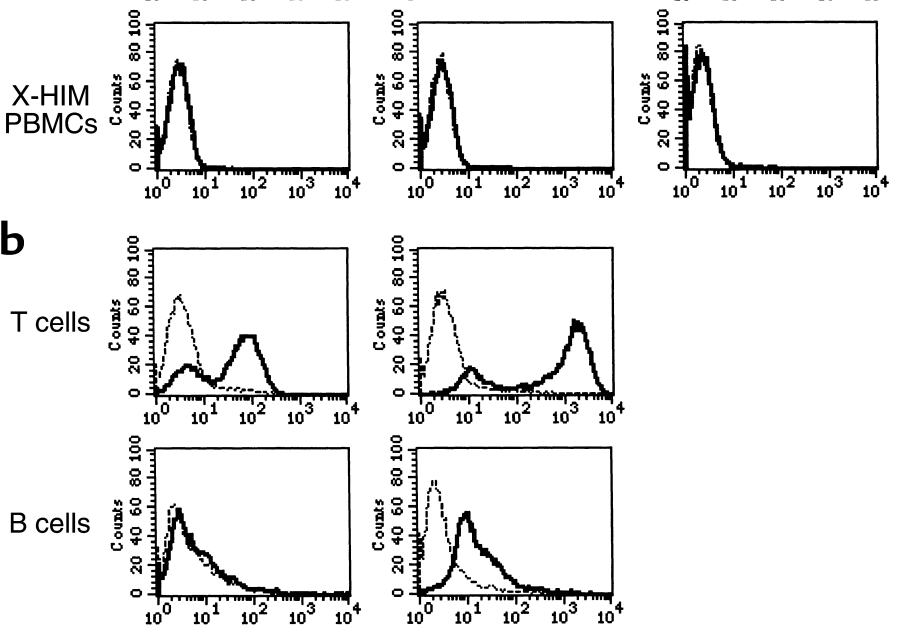

CD40L to their nuclear receptors. For instance, progesterone has been shown to bind to the oxytocin cell-surface receptor and potentiate the activity of oxytocin (32). To assess the requirement for GR in HC induction of CD40L expression and isotype switching, we examined the effect of the SHR antagonist RU-486. Figure 4a shows that addition of RU-486 $\left(10^{-6} \mathrm{M}\right)$ completely inhibited the upregulation of CD40L mRNA by HC. Figure $4 \mathrm{~b}$ shows that RU-486 inhibited induction of IgE synthesis by HC+IL-4 in a dose-dependent manner. This effect was specific because RU-486 did not inhibit the induction of IgE synthesis by anti-CD40+IL-4. RU-486 inhibits both GR and the progesterone receptor. However, since progesterone is unable to induce IgE synthesis in IL-4-stimulated PBMCs (ref. 22 and data not shown), these results suggest that GR is critical for upregulation of CD40L mRNA and for induction of IgE synthesis by HC.

\section{Discussion}

The results in this study show that induction of IgE isotype switching in human lymphocytes by the glucocorticoid HC is dependent on CD40L-CD40 interactions. $\mathrm{HC}$ was shown to upregulate CD40L expression by human PBMCs and purified populations of $\mathrm{T}$ and $B$ cells in a GR-dependent manner.

A critical role for CD40L-CD40 interactions in HC induction of $\operatorname{IgE}$ synthesis was demonstrated by the study of patients with CD40L deficiency. PBMCs from all five patients examined failed to synthesize $\operatorname{IgE}$ in response to HC+IL-4 (Table 1). This failure was clearly not due to intrinsic defects in IgE isotype switching by the patients' B cells, because their PBMCs synthesized IgE in response to two T-cell independent stimuli, namely anti-CD40 mAb and EBV. The normal response of $B$ cells from the patients to EBV indicates that induction of isotype switching by EBV is inde-

\section{Figure 4}

RU-486 inhibits upregulation of CD40L mRNA and induction of IgE synthesis. (a) Effect of RU-486 on CD40L mRNA expression is shown. PBMCs were left unstimulated or were stimulated with $\mathrm{HC}\left(10^{-6} \mathrm{M}\right)$ for 3 hours in the presence or absence of RU-486 $\left(10^{-6} \mathrm{M}\right)$. Values represent the mean plus or minus SE of the ratio of the intensity of the bands representing the RT-PCR products of CD40L and GAPDH in three experiments. (b) Effect of RU-486 on IgE synthesis. PBMCs were stimulated as described in Table 1 in the presence or absence of various concentrations of RU-486. Values represent the mean of net IgE synthesis measured in duplicate-culture supernatants at day 14 .
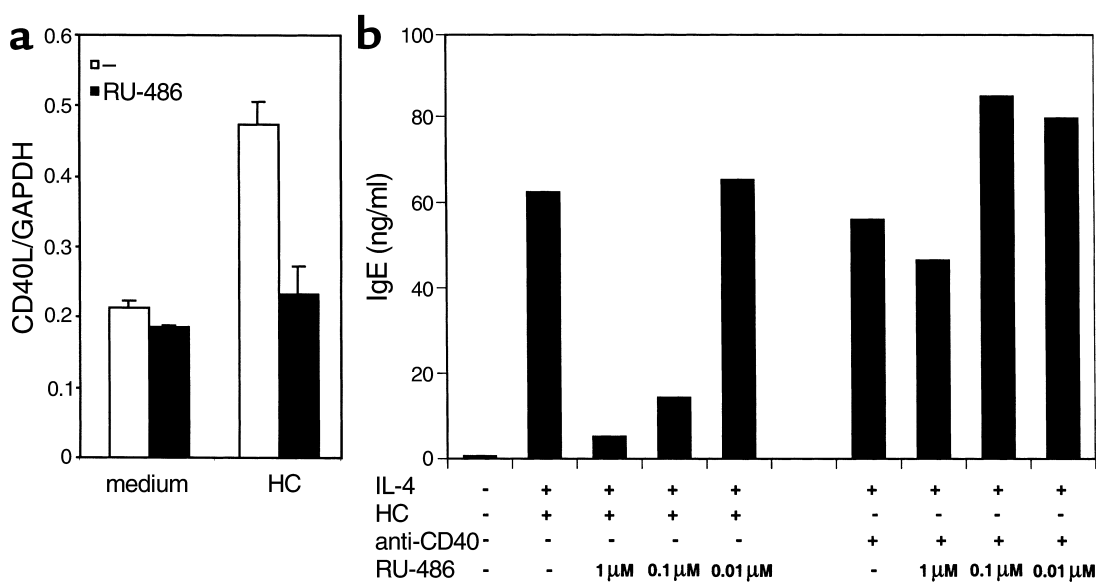
pendent of CD40L-CD40 interactions. This is consistent with recent findings, which suggest that isotype switching induced by EBV is mediated by the virus latent infection membrane protein-1 (LMP-1). Introduction of a transgene encoding for LMP-1 into $\mathrm{CD} 40^{-/-}$mice corrects the isotype switch defect in these mice (33). The intracellular tail of LMP-1 has homology to the intracellular domain of CD40 and, like CD40, associates with TNF receptor-associated factors and with Jak3 (34). Thus, expression of LMP-1, which oligomerizes spontaneously in the B-cell membrane may mimic CD40 ligation.

A role of CD40L-CD40 interactions in HC induction of isotype switching was established in normal cells using reagents that specifically block this interaction. Both sCD40-IgG fusion protein and the anti-CD40L $\mathrm{mAb} 5 \mathrm{c} 8$ block $\mathrm{T}$ cell-driven CD40L-dependent isotype switching by normal $\mathrm{B}$ cells $(8,30)$. Both reagents were found to block HC induction of IgE switching (Figure 1). These results indicate that CD40L-CD40 interactions are critical for $\mathrm{HC}$ induction of isotype switching.

HC was found to upregulate CD40L mRNA expression (Figure 2a). This effect was GC specific because it was observed with DM, but not with progesterone. Furthermore, it was likely due to enhanced CD40L gene transcription rather than to a stabilization of pre-existing CD40L mRNA, which was detectable in unstimulated cells, because actinomycin D completely abolished upregulation of CD40L mRNA by HC (Figure $2 \mathrm{~b})$. Induction of CD $40 \mathrm{~L}$ mRNA by $\mathrm{HC}$ was lower and less sustained than induction by PMA+IO (Figure 2, $\mathrm{c}$ and d). More importantly, $\mathrm{HC}$ induced expression of CD40L protein on the surface of PBMCs as detected by FACS analysis (Figure 3). This was difficult to detect using standard staining methods, but was readily detectable using enzymatic amplification. Very little expression of surface CD40L may be needed to induce isotype switching. IL-4, which is well known to induce CD40L-dependent IgE isotype switching in PBMCs (8), fails to induce detectable surface CD40L expression by conventional staining. However, when assessed by enzymatically enhanced flow cytometry, IL-4 causes upregulation of CD40L expression comparable to that caused by HC (data not shown).

HC induced CD40L expression in both highly purified populations of $\mathrm{T}$ and $\mathrm{B}$ cells. The ability of $\mathrm{HC}$ to induce CD40L expression in B cells may well explain our previous finding that $\mathrm{HC}$ induces IgE isotype switching in highly purified human B cells in the presence of IL-4 (21).

Use of enhanced flow cytometry revealed a baseline low level of CD40L expression on PBMCs. This is consistent with the detection in unstimulated PBMCs of CD40L mRNA by using RT-PCR (Figure 2a). Baseline CD40L expression is likely to reflect baseline expression of CD40L by $\mathrm{T}$ cells, since only purified T cells, but not purified $\mathrm{B}$ cells, had detectable baseline CD40L surface expression.

GCs are known to upregulate gene expression by interacting with GR and causing its translocation to the nucleus. We have established clearly that GR is involved in the upregulation of CD40L mRNA expression by HC because the SHR antagonist RU-486 inhibited HC upregulation of CD40L mRNA (Figure 4a) and induction of IgE synthesis (Figure 4b). Several potential mechanisms may be involved in upregulation of CD40L mRNA expression by GR. Ligand-bound GR may interact directly with putative GREs in the promoter region of the CD40L gene. Alternatively, GR may induce the expression of genes, the products of which may in turn upregulate CD40L gene expression. This is unlikely, as upregulation of CD40L mRNA expression by $\mathrm{HC}$ was insensitive to the protein synthesis inhibitor cycloheximide (data not shown). Finally, GR may enhance the baseline NF-AT/AP-1 complex activity to drive increased CD40L gene transcription.

Although the promoters of the human CD40L and IL-2 genes share several features including binding sites for AP-1, NF-AT, and NF-KB, GCs have an opposite effect on the expression of these two genes, i.e., suppression of IL-2 (35) and induction of CD40L (Figures 2 and 3). This suggests that GCs target elements in the CD40L promoter that are not present in the IL-2 gene promoter. Good candidates are GREs. The consensus sequence for GREs is AGAACANNNTGTTCT, with the nucleotides in boldface being almost invariant. Each of two GRs normally bind to each half palindromic site and dimerize to create a transcriptionally active complex that associates with coactivator proteins, e.g., p300 and CBP, and with TFIID (36). In some promoters, a single GR molecule binds to a sequence that contains only one half palindromic site and cooperates with adjacently bound transcription factors, e.g., STAT5, to drive transcription (37). Examination of the approximately $2-\mathrm{kb}$ upstream region of the human CD40L gene reveals five potential GRE full or half sites at -694 to $-713 \mathrm{bp},-1281$ to -1298 bp, -1513 to $-1530 \mathrm{bp},-1615$ to $-1634 \mathrm{bp}$, and -1646 to $-1665 \mathrm{bp}$. Preliminary data from electromobility shift assays show that GR binds to at least one of these sites (data not shown). Experiments are in progress to define the role of these sites in HC upregulation of CD40L gene expression.

The transcription factor NF-AT is activated by the calcium-dependent tyrosine phosphatase calcineurin, forms a transcriptionally active complex with AP-1, and plays a critical role in the induction of CD40L expression $(38,39)$. Baseline CD40L expression may reflect baseline NF-AT activity. GR may synergize with this baseline NF-AT activity to upregulate CD40L expression. This is suggested by our observations that the calcineurin inhibitor cyclosporin A (CsA), inhibits both baseline and $\mathrm{HC}$-induced CD40L mRNA expression (data not shown), although we cannot rule out effects of CsA that are independent of NF-AT. An enhancing effect of GR on NF-AT activity may be due to cooperative functional interactions between GR bound to sequences in the CD40L promoter and baseline NF-AT complexes bound to NF-AT sites in the 
CD40L promoter. Alternatively, GR may directly bind to NF-AT complexes and enhance their activity. This binding could involve the Jun component of AP-1, but also possibly NF-AT, as has been recently shown in the IL-4 promoter (40). Experiments are in progress to test these possibilities.

In addition to inducing IgE synthesis in vitro, administration of GCs has been reported to result in a significant rise in serum $\operatorname{IgE}$ in patients with asthma but not in normal nonatopic subjects $(41,42)$. This discrepancy may reflect the fact that induction of IgE synthesis by GCs requires IL-4, and IL-4 is detected in cells and serum of atopic patients but not of nonatopic controls (43-47). Despite their propensity to increase IgE secretion, GCs are effective in treating allergic diseases. This is likely due to their ability to downregulate the synthesis of T-cell cytokines that play a more critical role in allergic inflammation than IgE. This is evidenced by the observations that IgE-deficient mice develop allergic airway inflammation and allergic contact dermatitis to the same extent as wild-type controls $(48,49)$.

In addition to induction of isotype switching in $\mathrm{B}$ cells, CD40L activates a number of CD40-expressing inflammatory cells, including macrophages, dendritic cells, and eosinophils, and contributes to the elimination of pathogenic organisms. The importance of CD40L in the elimination of organisms is illustrated by the susceptibility of CD40L-deficient patients to Pneumocystis carinii pneumonia (50) and by the observation that exogenous $\mathrm{CD} 40 \mathrm{~L}$ is required for resolution of $P$. carinii pneumonia in scid mice (51). The concentration of $\mathrm{HC}$ that results in optimal CD40L expression $\left(10^{-6} \mathrm{M}\right)$ is in the range of the serum cortisol levels achieved in humans under stress conditions. Rapid induction of CD40L expression by GCs secreted in response to stress may be advantageous for a host upon first encounter with an infectious organism, when antigen-specific $\mathrm{T}$ cells have not yet expanded.

\section{Acknowledgments}

This work was supported by United States Public Health Service grants AI-31541 and AI-31136 and by the Baxter, Centeon and Olsten Corporations. S. Brodeur was supported by NIH training grant AI-07512.

1. Vercelli, D., and Geha, R.S. 1992. Regulation of isotype switching. Curr. Opin. Immunol. 4:794-799.

2. Zurawski, S., et al. 1995. The primary binding subunit of the human interleukin-4 receptor is also a component of the interleukin-13 receptor. J. Biol. Chem. 270:13869-13878.

3. Defrance, T., et al. 1994. Interleukin 13 is a B cell stimulating factor. J. Exp. Med. 179:135-143.

4. Vercelli, D., and Geha, R.S. 1993. Regulation of IgE synthesis: from the membrane to the genes. Springer Semin. Immunopathol. 15:5-16.

5. Shapira, S., Vercelli, D., Jabara, H., Fu, S., and Geha, R. 1992. Molecular analysis of the induction of immunoglobulin $\mathrm{E}$ synthesis by human $\mathrm{B}$ cells by interleukin 4 and engagement of CD40 antigen. J. Exp. Med. 175:289-292.

6. Muramatsu, M., et al. 1999. Specific expression of activation-induced cytidine deaminase (AID), a novel member of the RNA-editing deaminase family in germinal center B cells. J. Biol. Chem. 274:18470-18476.

7. Jabara, H., Fu, S., Geha, R., and Vercelli, D. 1990. CD40 and IgE: synergism between anti-CD40 monoclonal antibody and interleukin 4 in the induction of IgE synthesis by highly purified human B cells. J. Exp. Med. 172:1861-1864.
8. Fanslow, W., et al. 1992. Soluble forms of CD40 inhibit biological responses of human B cells. J. Immunol. 149:655-660.

9. Fuleihan, R., Ramesh, N., and Geha, R.S. 1995. X-linked agammaglobulinemia and immunoglobulin deficiency with normal or elevated IgM: immunodeficiencies of B cell development and differentiation. Adv. Immunol. 60:37-56.

10. Xu, J., et al. 1994. Mice deficient for CD40 ligand. Immunity. 1:423-431.

11. Castigli, E., et al. 1994. CD40-deficient mice generated by recombinationactivating gene-2-deficient blastocyst complementation. Proc. Natl. Acad. Sci. USA. 91:12135-12139.

12. Kawabe, T., et al. 1994. The immune responses in CD40-deficient mice: impaired immunoglobulin class switching and germinal center formation. Immunity. 1:167-178.

13. Beato, M., Herrlich, P., and Schutz, G. 1995. Steroid hormone receptors: many actors in search of a plot. Cell. 83:851-857.

14. Jonat, C., et al. 1990. Antitumor promotion and antiinflammation: downmodulation of AP-1 (Fos/Jun) activity by glucocorticoid hormone. Cell. 62:1189-1204.

15. Yang-Yen, H.-F., et al. 1990. Transcriptional interference between c-Jun and the glucocorticoid receptor: mutual inhibition of DNA binding due to direct protein-protein interaction. Cell. 62:1205-1215.

16. Stoecklin, E., Wissler, M., Moriggl, R., and Groner, B. 1997. Specific DNA binding of Stat5, but not of glucocorticoid receptor, is required for their functional cooperation in the regulation of gene transcription. Mol. Cell. Biol. 17:6708-6716.

17. Cella, N., Groner, B., and Hynes, N.E. 1998. Characterization of Stat5a and Stat $5 \mathrm{~b}$ homodimers and heterodimers and their association with the glucocorticoid receptor in mammary cells. Mol. Cell. Biol. 18:1783-1792.

18. Zhang, Z., Jones, S., Hagood, J.S., Fuentes, N.L., and Fuller, G.M. 1997. STAT3 acts as a co-activator of glucocorticoid receptor signaling. J. Biol. Chem. 272:30607-30610.

19. Prefontaine, G.G., et al. 1998. Recruitment of octamer transcription factors to DNA by glucocorticoid receptor. Mol. Cell. Biol. 18:3416-3430.

20. Aurade, F., et al. 1997. The glucocorticoid receptor and AP-1 are involved in a positive regulation of the muscle regulatory gene myf5 in cultured myoblasts. J. Cell Sci. 110:2771-2779.

21. Jabara, H., Ahern, D., Vercelli, D., and Geha, R. 1991. Hydrocortisone and IL-4 induce IgE isotype switching in human B cells. J. Immunol. 147:1557-1560.

22. Wu, C.Y., et al. 1991. Glucocorticoids increase the synthesis of immunoglobulin E by interleukin 4-stimulated human lymphocytes. J. Clin. Invest. 87:870-877.

23. Jabara, H., Loh, R., Ramesh, N., Vercelli, D., and Geha, R. 1993. Sequential switching from $\mu$ to $\varepsilon$ via $\gamma 4$ in human B cells stimulated with IL-4 and hydrocortisone. J. Immunol. 151:4528-4533.

24. Ramesh, N., et al. 1993. Deletions in the ligand for CD40 in X-linked immunoglobulin deficiency with normal or elevated IgM (HIGMX-1). Int. Immunol. 5:769-773.

25. Chu, Y.W., et al. 1995. Somatic mutation of human immunoglobulin V genes in the X-linked HyperIgM syndrome. J. Clin. Invest. 95:1389-1393.

26. Jabara, H., et al. 1990 . Induction of germ-line and mature $C \varepsilon$ transcripts in human B cells stimulated with rIL-4 and EBV. J. Immunol. 145:3468-3473.

27. Fuleihan, R., et al. 1993. Defective expression of the CD40 ligand in X chromosome-linked immunoglobulin deficiency with normal or elevated IgM. Proc. Natl. Acad. Sci. USA. 90:2170-2173.

28. Thienes, C., et al. 1997. The transcription factor B cell-specific activator protein (BSAP) enhances both IL-4- and CD40-mediated activation of the human $\varepsilon$ germline promoter. J. Immunol. 158:5874-5882.

29. Kaplan, D., and Smith, D. 2000. Enzymatic amplification staining for flow cytometric analysis of cell surface molecules. Cytometry. 40:81-85.

30. Jabara, H.H., and Geha, R.S. 1996. The superantigen toxic shock syndrome toxin- 1 induces CD40 ligand expression and modulates IgE isotype switching. Int. Immunol. 8:1503-1510.

31. Fuleihan, R., et al. 1994. Cyclosporin A inhibits CD40 ligand expression in T lymphocytes. J. Clin. Invest. 93:1315-1320.

32. Picard, D. 1998. Molecular endocrinology. Steroids tickle cells inside and out. Nature. 392:437-438.

33. Uchida, J., et al. 1999. Mimicry of CD40 signals by Epstein-Barr virus LMP1 in B lymphocyte responses. Science. 286:300-303.

34. Mosialos, G., et al. 1995. The Epstein-Barr virus transforming protein LMP-1 engages signaling proteins for the tumor necrosis factor receptor family. Cell. 80:389-399.

35. Northrop, J.P., Crabtree, G.R., and Mattila, P.S. 1992. Negative regulation of interleukin 2 transcription by the glucocorticoid receptor. J. Exp. Med. 175:1235-1245.

36. Horwitz, K.B., et al. 1996. Nuclear receptor coactivators and corepressors. Mol. Endocrinol. 10:1167-1177.

37. Lechner, J., Welte, T., and Doppler, W. 1997. Mechanism of interaction between the glucocorticoid receptor and Stat5: role of DNA-binding. Immunobiology. 198:112-123.

38. Tsytsykova, A., Tsitsikov, E., Ramesh, N., and Geha, R.S. 1996. The CD40L 
promoter contains nuclear factor of activated $\mathrm{T}$ cells-binding motifs which require AP-1 binding for activation of transcription. J. Biol. Chem. 271:3763-3770.

39. Schubert, L.A., et al. 1995. The human gp39 promoter. Two distinct nuclear factors of activated $\mathrm{T}$ cell protein-binding elements contribute independently to transcriptional activation. J. Biol. Chem. 270:29624-29267.

40. Chen, R., et al. 2000. Glucocorticoids inhibit calcium- and calcineurindependent activation of the human IL-4 promoter. J. Immunol. 164:825-832.

41. Zieg, G., Lack, G., Harbeck, R.J., Gelfand, E.W., and Leung, D.Y. 1994. In vivo effects of glucocorticoids on IgE production. J. Allergy Clin. Immunol. 94:222-230.

42. Klebl, F.H., Weber, G., Kalden, J.R., and Nusslein, H.J. 1994. In vitro and in vivo effects of glucocorticoids on $\operatorname{IgE}$ and $\operatorname{IgG}$ subclass secretion. Clin. Exp. Allergy. 24:1001-1004.

43. Esnault, S., et al. 1996. Differential spontaneous expression of mRNA for IL-4, IL-10, IL-13, IL-2 and interferon-gamma (IFN-gamma) in peripheral blood mononuclear cells (PBMC) from atopic patients. Clin. Exp. Immunol. 103:111-118.

44. Tang, M.L., and Kemp, A.S. 1994. Spontaneous expression of IL-4 mRNA in lymphocytes from children with atopic dermatitis. Clin. Exp. Immunol. 97:491-498.

45. Daher, S., et al. 1995. Interleukin-4 and soluble CD23 serum levels in asthmatic atopic children. J. Investig. Allergol. Clin. Immunol. 5:251-254.

46. Reddy, M.M., et al. 1992. Circulating elevated levels of soluble CD23, interleukin-4, and CD20+CD23+ lymphocytes in atopic subjects with elevated serum IgE concentrations. Ann. Allergy. 69:131-134.

47. Matsumoto, T., et al. 1991. Serum levels of soluble IL-2 receptor, IL-4 and IgE-binding factors in childhood allergic diseases. Clin. Exp. Immunol. 85:288-292.

48. Mehlhop, P.D., et al. 1997. Allergen-induced bronchial hyperreactivity and eosinophilic inflammation occur in the absence of $\operatorname{IgE}$ in a mouse model of asthma. Proc. Natl. Acad. Sci. USA. 94:1344-1349.

49. Spergel, J.M., Mizoguchi, E., Oettgen, H., Bhan, A.K., and Geha, R.S. 1999. Roles of TH1 and TH2 cytokines in a murine model of allergic dermatitis. J. Clin. Invest. 103:1103-1111.

50. Notarangelo, L.D., Duse, M., and Ugazio, A.G. 1992. Immunodeficiency with hyper-IgM (HIM). Immunodefic. Rev. 3:101-122.

51. Wiley, J.A., and Harmsen, A.G. 1995. CD40 ligand is required for resolution of Pneumocystis carinii pneumonia in mice. J. Immunol. 155:3525-3529. 